\title{
Risk Factor Assessment and a Ten-Year Experience of DDH Screening in a Well-Child Population
}

\author{
Bahar Kural (D), Esra Devecioğlu Karapınar, ${ }^{2}$ Pınar Yılmazbaş, \\ Tijen Eren, ${ }^{3}$ and Gülbin Gökçay ${ }^{4}$ \\ ${ }^{1}$ MD, Ph.D. Pediatrician, Istanbul University Institute of Child Health, Department of Social Pediatrics, Istanbul, Turkey \\ ${ }^{2} \mathrm{MD}$, Pediatrician, Istanbul University Institute of Child Health, Department of Social Pediatrics, Istanbul, Turkey \\ ${ }^{3}$ MD, Ph.D., Family Medicine Specialist, Istanbul University Institute of Child Health, Department of Social Pediatrics, Istanbul, Turkey \\ ${ }^{4}$ Professor of Pediatrics, Istanbul University Institute of Child Health, Department of Social Pediatrics, Istanbul, Turkey
}

Correspondence should be addressed to Bahar Kural; drbahsal@yahoo.com

Received 15 April 2019; Revised 15 July 2019; Accepted 18 July 2019; Published 4 August 2019

Academic Editor: Jonathan Muraskas

Copyright (C) 2019 Bahar Kural et al. This is an open access article distributed under the Creative Commons Attribution License, which permits unrestricted use, distribution, and reproduction in any medium, provided the original work is properly cited.

\begin{abstract}
Aim. Risk based screening for developmental dysplasia of the hip (DDH) with ultrasound is common. However, risk factors vary from one country to the other since data are insufficient to give clear recommendations. We aimed to evaluate the risk factors for developmental dysplasia of the hip (DDH). Methods. In this retrospective case-control study, the health records of all children, who were followed up between 2004 and 2014 at a well-child unit, were investigated for the diagnosis of DDH in Turkey. Of 9758 children, 57 children were found to have abnormal ultrasonographic findings (according to Graf classification) and these constituted the case group. As the control group, healthy 228 children who matched the case children in birth months were selected. Two groups were compared for the risk factors. Results. A total of 19516 hips of 9758 children were examined for DDH. 97 hips of 57 children were found to have abnormal ultrasonographic findings. When the two groups were compared, breech presentation, multiple pregnancy, and torticollis were identified as risk factors. The female sex was also found to have a significantly high prevalence among the children in the case group. Limited hip abduction, positive Ortolani, and Barlow signs were important clinical findings in the case group. Conclusion. According to our findings, breech presentation, female sex, torticollis, and multiple pregnancy were found to be the risk factors of this disorder. Infants with these risk factors should be investigated carefully for DDH.
\end{abstract}

\section{Introduction}

Screening programs for DDH have been present for many years. Different programs include pure clinical examination, selective ultrasonographic screening of at-risk newborns or universal neonatal ultrasonographic screening [1]. Age appropriate imaging is recommended by the American Academy of Pediatrics for female infants born breech or with family history of DDH [2]. In the United Kingdom, clinical hip instability in physical examination, family history of first degree relative requiring DDH treatment, breech position, multiple births if any of the babies is breech presentation are seen as risks requiring ultrasonographic investigation [3]. The screening of all newborns at birth for DDH using ultrasound imaging is standard practice in Germany, Austria, and Switzerland [4].
Risk based screening program was started in Turkey in 2013 as a pilot study and countrywide in 2014. All neonates with family history of DDH up to third-degree relatives, oligo/hydoamnios, breech presentation, foot deformities, plagiocephaly, scoliosis, congenital muscular torticollis (CMT), pelvic obliquity, adduction contracture of the hip, multiple pregnancy, and firstborn girl of the family are referred for ultrasound scanning [5].

Risk based screening for developmental dysplasia of the hip (DDH) with ultrasound is common in the world. On the other hand, risk factors vary from one country to the other since data are insufficient to give clear recommendations $[6$, 7].

In this retrospective case-control study, we aimed to investigate the risk factors for DDH in a well-child unit in Turkey. 
TABLE 1: Risk factors used for the screening of developmental dysplasia of the hip in the unit.

\begin{tabular}{l}
\hline Family history (first-degree relatives) \\
\hline Multiple pregnancy \\
\hline Breech presentation \\
\hline Oligohydramnios \\
\hline Congenital muscular torticollis \\
\hline Foot deformities \\
\hline Pathological clinical findings(Positive Ortolani and Barlow maneuvers, Positive Galeazzi sign, limited abduction, and asymmetric crease) \\
\hline
\end{tabular}

\section{Methods}

This retrospectively designed case-control study was carried out in the Well-Child Unit of Istanbul University Hospital in Turkey. Each child had a health record starting from 1 month of age at the unit. The health records of all children, who were followed between January 2004 and December 2014 were investigated for the diagnosis of DDH. Children admitted to the Unit include term infants born at the maternity clinic of the same hospital. Children were followed every 2 months for the first 6 months and every 3 months until 18 months of age and every 6 months thereafter until 10 years of age according to the program of Well-Child Unit. At each visit, detailed physical examination was carried out and findings were recorded in the personal files of the children. The basic sociodemographic information of each child was also kept. Physical examination and risk assessment for DDH were conducted for each infant starting at 1 month of age in the Unit. Barlow and Ortolani test were performed for this purpose until the third month of life. Asymmetric thigh or perineal crease, an apparent short leg (positive Galeazzi sign) and limitation of hip abduction were also sought as clinical findings. Children with risk factors listed in Table 1 underwent ultrasound examination at the unit. The walking pattern of the child was also evaluated during the visits. Detailed family, perinatal and natal history (including birth weight) were taken for each child at the first admission. According to the unit's policy, parents were guided about the clothing for the children and swaddling was not recommended. Sonographic examinations and classifications were performed in combination with the Graf technique [8]. The final combined clinical and sonographical examination for each child was carried out by a pediatrician and/or a pediatric resident under supervision of the senior author (GG) before the referral of the children to the orthopedic surgeon. Infants older than 3 months with abnormal ultrasonographic findings (Graf classification type IIa, IIc, and D,III/IV) constituted the case group [8]. Two children before and two after each case child as listed in the records, who were born in the same month and in the same year were selected as controls; therefore four controls were selected per case. The control group encompassed 228 children. The study design was given in Figure 1. All children in the study had been followed up until 18 months of age.

Ethical approval and necessary institutional permissions were obtained for the study.

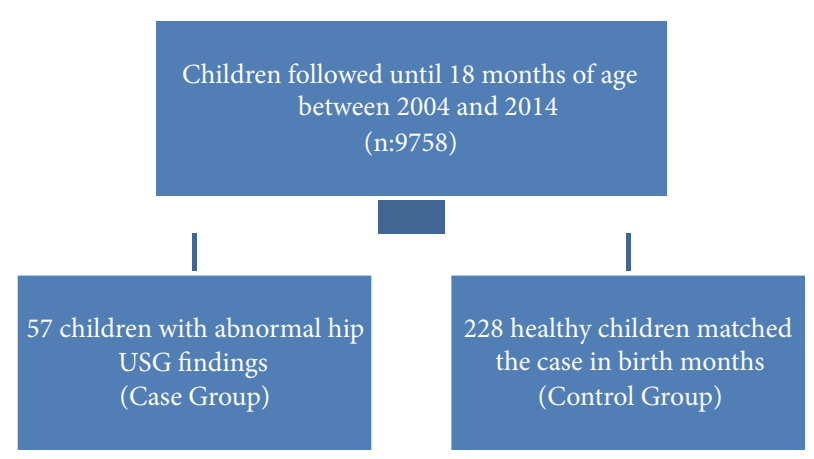

FIgure 1: The study design.

TABLE 2: Age distribution of the cases at the first hip ultrasonography (USG).

\begin{tabular}{lc}
\hline Timing of USG (weeks) & $\mathrm{n}(\%)$ \\
\hline 4 & $24(42,1)$ \\
\hline 6 & $5(8,8)$ \\
\hline 8 & $26(45,6)$ \\
\hline 12 & $1(1,8)$ \\
\hline Total & $1(1,8)$ \\
\hline
\end{tabular}

\section{Statistical Analyses}

The NCSS (Number Cruncher Statistical System) 2007 (Kaysville, UT, USA) program was used for the statistical analysis. Student's t test, Pearson $\chi^{2}$ test, and Fisher's exact test were carried out for the analysis. Results were evaluated at the $95 \%$ confidence interval $(95 \% \mathrm{CI})$ range and $\mathrm{p}<0.05$ significance level.

\section{Results}

Of the 9758 children, $57(0.58 \%)$ had abnormal ultrasonographic findings. The age distribution of the cases at the first ultrasonography is given in Table 2 . This study encompassed the sonographic findings of 97 hips in 57 infants.

The orthopedic treatment and follow-up data of 57 children were evaluated. The distribution of clinical findings and risk factors in case and control groups are presented in Table 3. In the case group, one child with arthrogryposis 
TABLE 3: Distribution of risk factors and clinical findings in the case and control groups $(n=285)$.

\begin{tabular}{|c|c|c|c|}
\hline & Case $n: 57 \mathrm{n}(\%)$ & Control n:228 n (\%) & p value \\
\hline Family history of DDH & $5(8.8)$ & $11(4.8)$ & ${ }^{b} 0.329$ \\
\hline Female sex & $40(70,2)$ & $116(50,9)$ & ${ }^{b} 0,009$ \\
\hline Breech delivery & $9(15.8)$ & $16(7.0)$ & ${ }^{a} 0.036$ \\
\hline Multiple pregnancy & $5(8.8)$ & $5(2.2)$ & ${ }^{b} 0.030$ \\
\hline Oligohydramnios & $1(1.8)$ & $8(3.5)$ & ${ }^{b} 0.693$ \\
\hline Ortolani maneuver positivity & $4(7.0)$ & 0 & 0.001 \\
\hline Barlow maneuver positivity & $3(5.3)$ & 0 & 0.008 \\
\hline Asymmetric thigh or perineal crease & $14(24.6)$ & $58(25.4)$ & ${ }^{a} 0.892$ \\
\hline Limited hip abduction & $27(47.4)$ & $6(2.6)$ & 0.001 \\
\hline Congenital muscular torticollis & $3(5.3)$ & $1(0.4)$ & ${ }^{b} 0.026$ \\
\hline Foot deformities & $2(3.5)$ & $2(0.9)$ & 0.180 \\
\hline
\end{tabular}

${ }^{a}$ Pearson $\chi^{2}$ test. ${ }^{b}$ Fisher's exact test.

TABLE 4: Distribution of risk factors in case group according to management.

\begin{tabular}{lccc}
\hline Risk factors & $\begin{array}{c}\text { Brace treatment } \\
(n: 19)\end{array}$ & $\begin{array}{c}\text { Follow-up without treatment }(n: 37) \\
\text { Operation } \\
(n: 1) *\end{array}$ & - \\
\hline Family history of DDH & 2 & 3 & 1 \\
\hline Female sex & 15 & 5 & - \\
\hline Breech delivery & 4 & 4 & - \\
\hline Multiple pregnancy & 1 & 1 & \\
\hline Oligohydramnios & - & 24 & - \\
\hline Ortolani maneuver positivity & 1 & 14 & - \\
\hline Barlow maneuver positivity & 1 & 8 & - \\
\hline Asymmetric thigh or perineal crease & - & - & - \\
\hline Limited hip abduction & 18 & - & 1 \\
\hline Congenital muscular torticollis & 3 & 2 & - \\
\hline Foot deformities & & & - \\
\hline
\end{tabular}

*: the patient had arthrogryposis multiplex.

multiplex had operation. An abduction brace such as a Pavlik harness was applied to 19 infants. As a result of this finding, true DDH was $0.2 \%$. The distribution of the risk factors in case group according to management protocol are provided in Table 4. Some children have more than one risk factor. The distribution of risk factors was similar. The 37 untreated infants were carefully followed clinically and sonographically. Repeated ultrasonographical and radiological examination of these infants were normal by the age of 18 months.

Results of unilateral analysis of risk factors which were statistically significant are given in Table 5. Our results showed a statistically significant difference in the proportion of females versus males, who were at risk for developing $\mathrm{DDH}$; female children had 2.27 times greater odds of DDH risk than male children. Cases had three times greater odds of breech presentation and eight times greater odds of CMT and four times greater odds of multiple pregnancy than children in the control group.

Limited hip abduction was significantly higher in the case group than in the control group. Unilateral limitation of abduction had a positive predictive value of $82 \%$. The Galeazzi sign was not noted in both groups.
All children were followed-up until the walking age and no late case was identified.

\section{Discussion}

A 10-year DDH risk-based screening experience was presented in this study. Breech presentation, female sex, torticollis, and multiple pregnancy were found to be the statistically significant risk factors. The significant findings were the Ortolani and Barlow maneuvers and limitation of hip abduction.

The early identification of children with $\mathrm{DDH}$ is valuable as it allows for less invasive corrective procedures than if $\mathrm{DDH}$ is identified late [9]. In our study, the oldest age for diagnosis of DDH was 4 months.

Peled et al. defined "true DDH" as a hip with a subsequent treatment and that definition lowered the incidence to $0.5 \%$ [10]. According to this definition, we had only $20(0.2 \%)$ children with treatment (surgery or brace). Studies from Turkey have revealed a wide range of $0.5-28.1 \%$ depending on screening method, definition and population [11, 12]. 
TABLE 5: Results of unilateral analysis of risk factors.

\begin{tabular}{lcc}
\hline Risk Factor & Odds Ratio (OR) & Confidence Interval (CI) \\
\hline Female sex & 2.27 & $1.21-4.24$ \\
\hline Breech delivery & 2.96 & $1.25-7.04$ \\
\hline Multiple pregnancy & 3.83 & $1.17-12.53$ \\
\hline Congenital muscular torticollis & 7.88 & $1.28-48.33$ \\
\hline Limited hip abduction & 33.3 & $12.7-87.25$ \\
\hline
\end{tabular}

Lipton et al. investigated hips that showed a positive Ortolani sign on ultrasound and concluded that this sign indicated an abnormal ultrasonographic finding [13]. Choudry et al. suggested that limitation of hip abduction should be actively sought after 8 weeks of age and if present, ultrasonographic or radiographic examination should be performed [14]. Roposch et al. showed that even among pediatric orthopedic surgeons, there were wide variations in the diagnostic criteria for DDH in infants [15]. In our study, all children with positive Ortolani and Barlow maneuvers were in the case group. Unilateral limitation of abduction had a positive predictive value of $82 \%$ in the group.

In a Dutch study, total of 683 babies between 3 to 10 months old, limitation of abduction had a positive predictive value of $43 \%$ [16]. Our findings revealed that in the unit, clinical screening of DDH was successfully carried out by the physicians.

In our study, a family history of DDH was not found to be a risk factor. This may be because of our limited definition of "family history". The definition of family history in the literature ranges from unspecified hip disorders to hip dislocation and from first-degree relatives (parents and siblings) to any relative (even if distant or vague) with hip problems or DDH [17]. As we evaluated the health records retrospectively, we could not extend the definition of family history. Recent studies suggested that history of DDH and hip osteoarthritis among any family members should be considered as risk factor [18].

Female sex -whether in a first-born or not-is a wellknown risk factor for DDH, probably because of increased ligamentous laxity due to the circulating maternal hormone relaxin [19]. In our study, the risk in females was identified to be 2.27 times higher than that in males. In a meta-analysis of 31 studies, the relative ratio (RR) in newborn females was found to be 2.54 (95\% CI: 2.11-3.05) times more prevalent than in males [20].

The definition of oligohydramnios has changed over time with the use of ultrasonography $[21,22]$. Therefore, there are conflicting results in the literature about oligohydramnios as a risk factor for DDH[22]. In a study, Paton stated that oligohydramnios did not appear to be true risk factor in the development of pathological DDH [23]. In our study, it was also not found to be a risk factor for DDH.

Findings about multiple pregnancy as a risk factor for $\mathrm{DDH}$ are also controversial. Some authors did not recommend routine ultrasound screening for twins and triplets [24-26]. In our study, multiple pregnancy (twins or triplets) was found to be a risk factor for $\mathrm{DDH}$. This may be due to the additional risk factors of multiple pregnancy [24].

The frequency of DDH among children with breech presentation was reported to be between $17-23 \%$ [27]. It has been considered the most important environmental factor for $\mathrm{DDH}$ [22]. In our study, breech presentation was confirmed as a risk factor and $36 \%$ of all children (cases and controls) with breech had DDH. The risk of DDH is the highest in frank breech presentation (one or both knees extended) [28]. In our study, breech history was obtained from patients' files and there was no detailed types of breech presentation.

There is no consensus on the routine hip imaging screening of patients with congenital muscular torticollis [17, 29, 30]. In our study, torticollis seemed to be a risk factor for DDH. All CMT cases with DDH received brace treatment. Our finding led us to think that an ultrasonographic evaluation of the hips should be carried out for children with CMT.

Our study had some limitations. First, as we mentioned above, our definition of family history was limited in this study. Second, preterm infants were not included. Finally, we did not gather information about swaddling. Further prospective studies that investigate these factors are needed to confirm our findings.

\section{Conclusion}

Our study had important findings about the risk factors for DDH. The results showed that physical examination is still an important tool in the screening. Breech presentation, female sex, torticollis, and multiple pregnancy seemed to be risk factors for this disorder and infants at risk should be investigated carefully by means of ultrasonography.

\section{Data Availability}

The data used to support the findings of this study are available from the corresponding author upon request.

\section{Disclosure}

The study was conducted in Istanbul University, Istanbul Medical Faculty Hospital, Department of Pediatrics, WellChild Follow-Up Clinic. In this retrospective case control study, nearly 10.000 infants, who were followed up within 10 years, were investigated. The health records (files) of 
all children, who were followed between January 2004 and December 2014 were investigated for the diagnosis of $\mathrm{DDH}$.

\section{Conflicts of Interest}

The authors declare that no conflicts of interest exist.

\section{References}

[1] L. B. Laborie, I. O. Engesaeter, T. G. Lehmann, D. M. Eastwood, L. B. Engesaeter, and K. Rosendahl, "Screening strategies for hip dysplasia: long-term outcome of a randomized controlled trial," Pediatrics, vol. 132, no. 3, pp. 492-501, 2013.

[2] D. E. Bloomfield, E. A. Dinolfo, and F. Kokotas, "Care of the newborn after delivery," in American Academy of Pediatrics Textbook of Pediatric Care, T. McInery, Ed., pp. 742-749, American Academy of Pediatrics, Elk Grove Village IL, USA, 2nd edition, 2017.

[3] Public Health England, Newborn and Infant Physical Examination Screening Programme Standards 2016/17, 2016, https://www .gov.uk/government/uploads/system/uploads/attachment_data/ file/524424/NIPE_Programme_Standards_2016_to_2017.pdf.

[4] N. F. Woolacott, M. A. Puhan, J. Steurer, and J. Kleijnen, "Ultrasonography in screening for developmental dysplasia of the hip in newborns: Systematic review," British Medical Journal, vol. 330, no. 7505, pp. 1413-1415, 2005.

[5] Turkish Public Health Institution-Children and Adolescent Health Department, Infant, Toddler and Adolescent Monitoring Protocols, https://dosyaism.saglik.gov.tr/Eklenti/9010,bebek-cocuk-izlem-protokolleripdf.pdf?

[6] D. Shorter, T. Hong, and D. A. Osborn, "Cochrane review: screening programmes for developmental dysplasia of the hip in newborn infants," Evidence-Based Child Health, vol. 8, no. 1, pp. 11-54, 2013.

[7] M. D. Sewell and D. M. Eastwood, "Screening and treatment in developmental dysplasia of the hip-Where do we go from here?" International Orthopaedics, vol. 35, no. 9, pp. 1359-1367, 2011.

[8] R. Graf, C. Tschauner, and W. Klapsch, "Progress in prevention of late developmental dislocation of the hip by sonographic newborn hip "screening": Results of a comparative follow-up study," Journal of Pediatric Orthopaedics B, vol. 2, no. 2, pp. 115121, 1993.

[9] J. S. Sampath, S. Deakin, and R. W. Paton, "Splintage in developmental dysplasia of the hip: how low can we go?" Journal of Pediatric Orthopaedics, vol. 23, no. 3, pp. 352-355, 2003.

[10] E. Peled, M. Eidelman, A. Katzman, and V. Bialik, "Neonatal incidence of hip dysplasia," Clinical Orthopaedics and Related Research, vol. 466, no. 4, pp. 771-775, 2008.

[11] O. Guler, A. Şeker, S. Mutlu, M. H. Çerçi, and B. Kömür, "Results of a universal ultrasonographic hip screening program at a single institution," Acta Orthopaedica et Traumatologica Turcica, vol. 50, no. 1, pp. 42-48, 2016.

[12] P. Koşar, E. Ergun, H. Yiğit, F. D. Gökharman, and U. Koşar, "Developmental dysplasia in male infants: risk factors, instability and ultrasound screening," Hip International, vol. 21, no. 4, pp. 409-414, 2018.

[13] G. E. Lipton, J. T. Guille, H. Altiok, J. R. Bowen, and H. T. Harcke, "A reappraisal of the Ortolani examination in children with developmental dysplasia of the hip," Journal of Pediatric Orthopaedics, vol. 27, no. 1, pp. 27-31, 2007.
[14] Q. Choudry, R. Goyal, and R. W. Paton, "Is limitation of hip abduction a useful clinical sign in the diagnosis of developmental dysplasia of the hip?" Archives of Disease in Childhood, vol. 98, no. 11, pp. 862-866, 2013.

[15] A. Roposch, L. Q. Liu, and E. Protopapa, "Variations in the use of diagnostic criteria for developmental dysplasia of the hip," Clinical Orthopaedics and Related Research, vol. 471, no. 6, pp. 1946-1954, 2013.

[16] R. Castelein and J. Korte, "Limited hip abduction in the infant," Journal of Pediatric Orthopaedics, vol. 21, no. 5, pp. 668-670, 2001.

[17] K. Mulpuri, K. M. Song, M. J. Goldberg, and K. Sevarino, "Detection and nonoperative management of pediatric developmental dysplasia of the hip in infants up to six months of age," Journal of the American Academy of OrthopaedicSurgeons, vol. 23, no. 3, pp. 202-205, 2015.

[18] E. L. Sink, B. F. Ricciardi, K. D. Torre, and C. T. Price, "Selective ultrasound screening is inadequate to identify patients who present with symptomatic adult acetabular dysplasia," Journal of Children's Orthopaedics, vol. 8, no. 6, pp. 451-455, 2014.

[19] P. Kotlarsky, R. Haber, V. Bialik, and M. Eidelman, "Developmental dysplasia of the hip: What has changed in the last 20 years?" World Journal of Orthopedics, vol. 6, no. 11, pp. 886-901, 2015.

[20] C. L. Ortiz-Neira, E. O. Paolucci, and T. Donnon, "A metaanalysis of common risk factors associated with the diagnosis of developmental dysplasia of the hip in newborns," European Journal of Radiology, vol. 81, no. 3, pp. e344-e351, 2012.

[21] W. Moh, J. M. Graham Jr., I. Wadhawan, and P. A. SanchezLara, "Extrinsic factors influencing fetal deformations and intrauterine growth restriction," Journal of Pregnancy, vol. 2012, Article ID 750485, 11 pages, 2012.

[22] A. Chan, K. A. McCaul, P. J. Cundy, E. A. Haan, and R. ByronScott, "Perinatal risk factors for developmental dysplasia of the hip," ADC - Fetal and Neonatal Edition, vol. 76, no. 2, pp. F94F100, 1997.

[23] R. W. Paton, "Screening in developmental dysplasia of the hip (DDH)," The Surgeon, vol. 15, no. 5, pp. 290-296, 2017.

[24] M. De Pellegrin and D. Moharamzadeh, "Developmental dysplasia of the hip in twins: The importance of mechanical factors in the etiology of DDH," Journal of Pediatric Orthopaedics, vol. 30, no. 8, pp. 774-778, 2010.

[25] R. J. Bielski, M. W. Gesell, A. L. Teng, D. H. Cooper, and J. K. Muraskas, "Orthopaedic implications of multiple gestation pregnancy with triplets," Journal of Pediatric Orthopaedics, vol. 26, no. 1, pp. 129-131, 2006.

[26] L. V. Barr and A. Rehm, "Should all twins and multiple births undergo ultrasound examination for developmental dysplasia of the hip?" The Bone \& Joint Journal, vol. 95-B, no. 1, pp. 132134, 2013.

[27] E. J. Sarkissian, W. N. Sankar, K. Baldwin, and J. M. Flynn, "Is there a predilection for breech infants to demonstrate spontaneous stabilization of DDH instability?" Journal of Pediatric Orthopaedics, vol. 34, no. 5, pp. 509-513, 2014.

[28] H. Ömeroğlu, A. Akceylan, and N. Köse, "Associations between risk factors and developmental dysplasia of the hip and ultrasonographic hip type: a retrospective case control study," Journal of Children's Orthopaedics, vol. 13, no. 2, pp. 161-166, 2019.

[29] S. N. Kim, Y. B. Shin, W. Kim et al., "Screening for the coexistence of congenital muscular torticollis and developmental 
dysplasia of hip," Annals of Rehabilitation Medicine, vol. 35, no. 4, pp. 485-490, 2011.

[30] E. R. Joiner, L. M. Andras, and D. L. Skaggs, "Screening for hip dysplasia in congenital muscular torticollis: is physical exam enough?" Journal of Children's Orthopaedics, vol. 8, no. 2, pp. 115-119, 2014. 


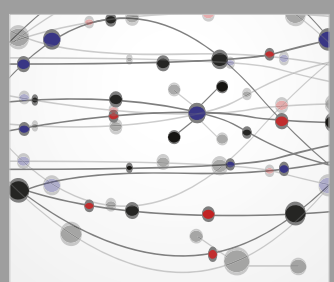

The Scientific World Journal
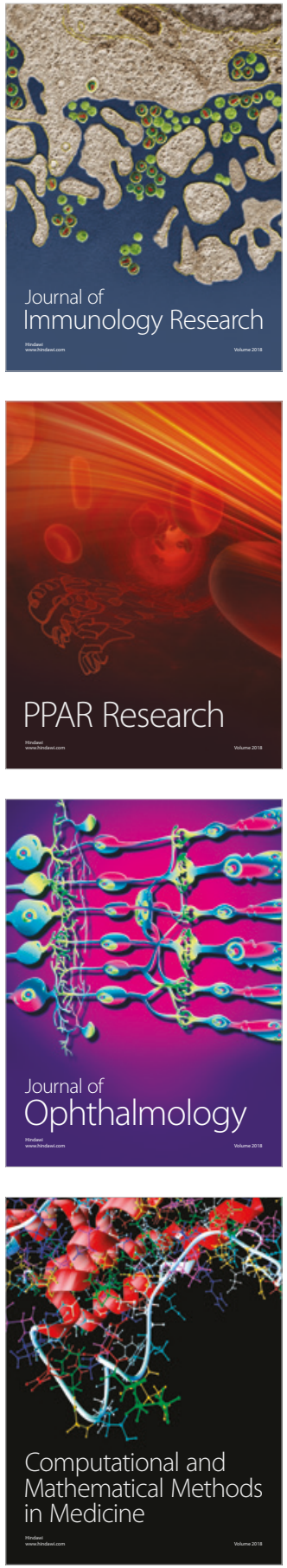

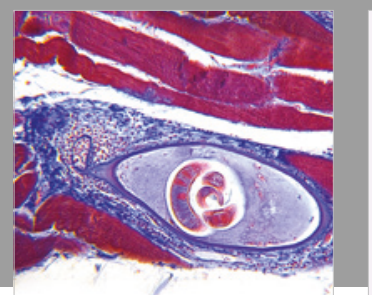

Gastroenterology Research and Practice

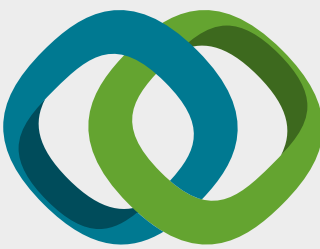

\section{Hindawi}

Submit your manuscripts at

www.hindawi.com
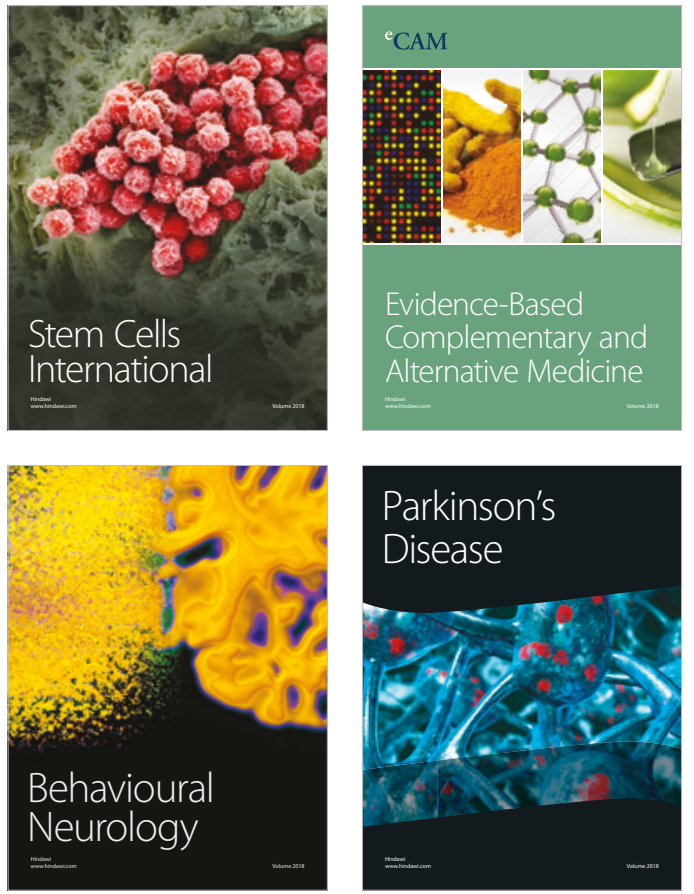

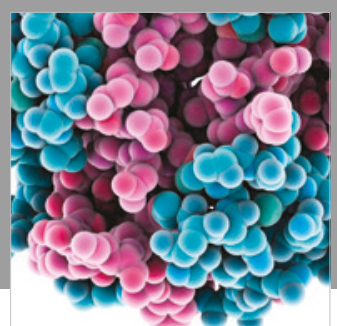

ournal of

Diabetes Research

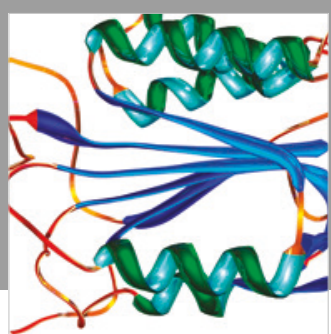

Disease Markers
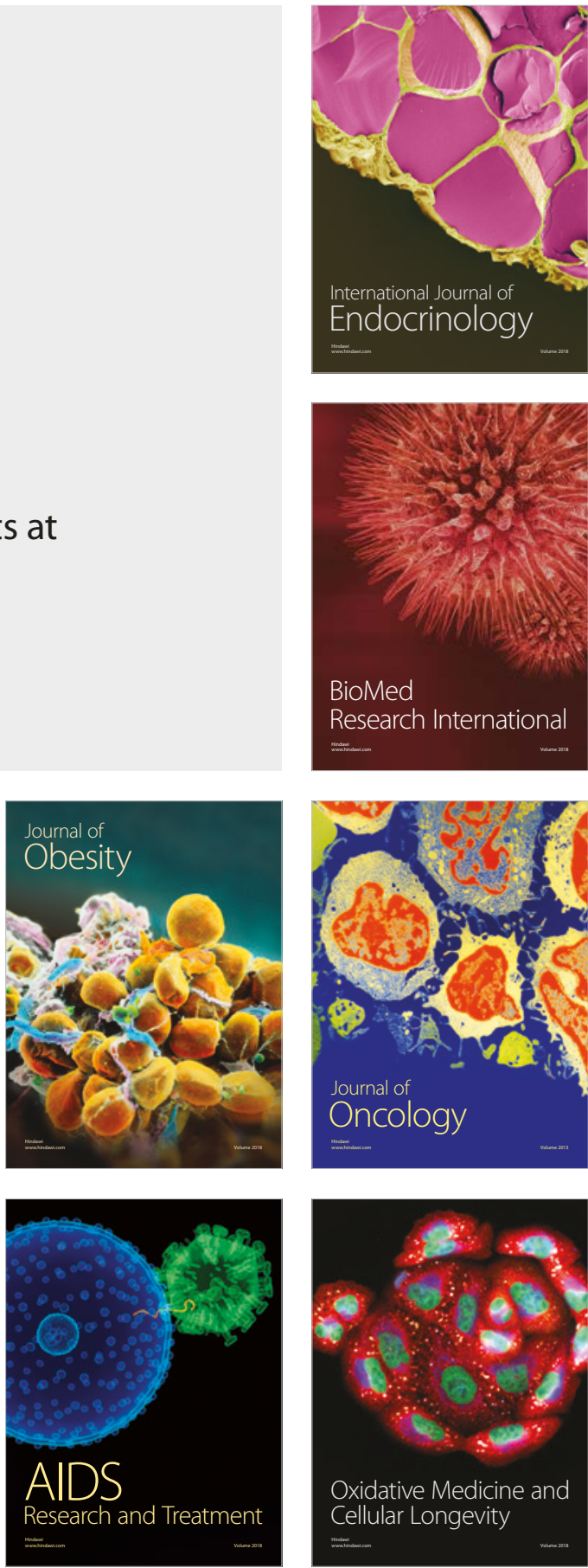